\title{
Applicable Multiplicative Calculus Using Multiplicative Modulus Function
}

\author{
C. Ganesa Moorthy \\ Department of Mathematics, Alagappa university, Karaikudi-630 003, India \\ Corresponding author E-mail: ganesamoorthyc@gmail.com
}

\author{
Article Info \\ Keywords: Differentiation, Positive \\ measure integration, Riemann integra- \\ tion \\ 2010 AMS: Primary 26A42; Secondary \\ 26A24; 26406. \\ Received: 07 October 2019 \\ Accepted: 18 December 2019 \\ Available online: 20 December 2019
}

\begin{abstract}
The classical calculus is viewed as additive calculus based on addition in the real line. Another known multiplicative calculus corresponding to multiplication in the positive real axis has been precisely introduced. Abstract multiplicative integration through positive measures has been newly introduced. Results of multiplicative differentiation and integration have been obtained for completion, when some of them have been obtained through multiplicative modulus function. Results have been obtained also for abstract multiplicative measure integration.
\end{abstract}

\section{Introduction}

Arithmetic mean of real numbers is considered in elementary statistics and geometric mean of positive real numbers is also considered in elementary statistics. The first one is a mean for addition and the second one is a mean for multiplication, and both are considered as applicable. The usual absolute value function is a function for addition. A new absolute value function, which was just mentioned in [1], has been extremely used for multiplication in [2]. The classical calculus of Newton and Leibnitz is based on addition. Another calculus was also known for multiplication, and it became an important part of research since the publication of the book [3] and the article [1], which provides a good introduction for multiplicative calculus. It has been extended in many directions; fractional derivative, complex derivative, integral transformations, differential equations and applications for science and engineering in [1], [4]-[18]. It has been established in $[19,20]$ that multiplicative calculus would also be applicable. The author believes that some precision is required in the introduction of multiplicative calculus, because it is also treated as a course meant for undergraduate students in view of articles like [21]. This has been done in this article. Moreover, multiplicative modulus function used in [2] has been applied to derive some new, but elementary results. Multiplicative integration using positive measures has also been defined precisely and some fundamental results have been obtained. The author could not find any article in literature for multiplicative abstract measure integration, even though there is an advanced research article [22] for Lebesgue measure integration. It should be observed that some changes have been done in this article in conventional notations for multiplicative calculus. Let us begin with a definition of the classical absolute value of real numbers. Let us use the notations $\mathrm{R}$ and $\mathrm{P}$ for the set of real numbers and the set of positive real numbers, respectively.

Definition 1.1. If $x \in R$, then additive absolute value of $x$ is denoted by $|x|$, and defined as the number max $\{x,-x\}$.

Let us now present the notation and definition of multiplicative absolute value given in [2].

Definition 1.2. If $x \in P$, then multiplicative absolute value of $x$ is denoted by $|x|_{\times}$, and defined as the number max $\left\{x, x^{-1}\right\}$.

Proposition 1.3. Let $x, y \in R$, and let $u, v \in P$. Then $|u v|_{\times} \leq|u|_{\times}|v|_{\times},|\log u|=\log |u|_{\times}$, and $\exp |x|=|\exp x|_{\times}$.

Proof. Direct verification.

The second section discusses fundamental results for multiplicative differentiation for completion, the third section discusses multiplicative Riemann integration with a precise definition, and the fourth section discusses newly introduced multiplicative abstract measure integration. 


\section{Multiplicative differentiation}

Definition 2.1. Let $f:[a, b] \rightarrow R$ be a real valued function and let $x_{0} \in[a, b]$. Then the derivative of $f$ exists at $x_{0}$, if $\lim _{x \rightarrow x_{0}} \frac{f(x)-f\left(x_{0}\right)}{x-x_{0}}$ exists. This limit is denoted by $f^{\prime}\left(x_{0}\right)$ or $\frac{d f\left(x_{0}\right)}{d x}$ or $\left.\frac{d f}{d x}\right|_{x=x_{0}}$ or $\left.\frac{d f(x)}{d x}\right|_{x=x_{0}}$, and it is called the derivative of $f$ at $x_{0}$.

Definition 2.2. Let $F:[a, b] \rightarrow(0, \infty)$ be a positive real valued function and let $x_{0} \in[a, b]$. Then the $m$-derivative of $F$ exists at $x_{0}$, if $\lim _{x \rightarrow x_{0}}\left(\frac{F(x)}{F\left(x_{0}\right)}\right)^{\frac{1}{x-x_{0}}}$ exists, and it is not equal to zero. This limit is denoted by $F^{\mid}\left(x_{0}\right)$ or $\frac{D F\left(x_{0}\right)}{D x}$ or $\left.\frac{D F}{D x}\right|_{x=x_{0}}$ or $\left.\frac{D F(x)}{D x}\right|_{x=x_{0}}$, and it is called the $m$-derivative of $F$ at $x_{0}$.

Remark 2.3. These definitions can be extended to other intervals of types $[a, b),(a, b),(a, b]$, naturally. One sided derivatives can also be defined. The higher order derivatives can also be defined.

Lemma 2.4. Let $F,[a, b], x_{0}$ be as in Definition 2.2. Then $\frac{D F\left(x_{0}\right)}{D x}$ exists if and only if $\frac{d \log F\left(x_{0}\right)}{d x}$ exists, and in this case, $\frac{D F\left(x_{0}\right)}{D x}=$ $\exp \left(\frac{d \log F\left(x_{0}\right)}{d x}\right)$.

Proof. The proof follows from the relation $\exp \left(\frac{\log F(x)-\log F\left(x_{0}\right)}{x-x_{0}}\right)=\left(\frac{F(x)}{F\left(x_{0}\right)}\right)^{\frac{1}{x-x_{0}}}$.

Corollary 2.5. $\frac{D F\left(x_{0}\right)}{D x}$ exists if and only if $\frac{d F\left(x_{0}\right)}{d x}$ exists, for $F$ and $x_{0}$ given in Lemma 2.4.

Proof. Use the relation $F(x)=\exp \log F(x)$, and the chain rule.

Lemma 2.6. Let $f,[a, b], x_{0}$ be as in Definition 2.1. Then $\frac{d f\left(x_{0}\right)}{d x}$ exists if and only if $\frac{D \exp \left(f\left(x_{0}\right)\right)}{D x}$ exists, and in this case, $\frac{d f\left(x_{0}\right)}{d x}=$ $\log \left(\frac{D \exp \left(f\left(x_{0}\right)\right)}{D x}\right)$.

Proof. The proof follows from the relation $\log \left(\frac{\exp (f(x))}{\exp \left(f\left(x_{0}\right)\right)}\right)^{\frac{1}{x-x_{0}}}=\frac{f(x)-f\left(x_{0}\right)}{x-x_{0}}$.

Remark 2.7. Formally, $\frac{D}{D x}=\exp \frac{d}{d x} \log$, and $\frac{d}{d x}=\log \frac{D}{D x} \exp$. For any integer $n \geq 2$, it can be verified formally that $\frac{D^{n}}{D x^{n}}=\exp \frac{d^{n}}{d x^{n}} \log$, and $\frac{d^{n}}{d x^{n}}=\log \frac{D^{n}}{D x^{n}} \exp$.

Let us try to use these formal relations and let us convert some results of Chapter 5 in [23].

Proposition 2.8. Let $F,[a, b], x_{0}$ be as in Definition 2.2. Suppose $\frac{D F\left(x_{0}\right)}{D x}$ exists. Then $F$ is continuous at $x_{0}$.

Proof. Let $M=\frac{D F\left(x_{0}\right)}{D x}$. Then $0<M<\infty$, and $M^{x-x_{0}} \rightarrow 1$ as $x \rightarrow x_{0}$. Therefore, $\frac{F(x)}{F\left(x_{0}\right)} \rightarrow 1$ and hence $F(x) \rightarrow F\left(x_{0}\right)$ as $x \rightarrow x_{0}$. Another Proof:

Since $\frac{d \log F\left(x_{0}\right)}{d x}$ exists, $\log F\left(x_{0}\right)$ is continuous at $x_{0}$, and hence $F(x)$ is continuous at $x_{0}$.

Proposition 2.9. Let $F, G$ be positive real valued functions on $[a, b]$ and $m$-differentiable at a point $x_{0}$ in $[a, b]$. Then $\frac{D(F G)\left(x_{0}\right)}{D x}$ exists and it is $\frac{D F\left(x_{0}\right)}{D x} \frac{D G\left(x_{0}\right)}{D x}$.

Proof. It follows from Definition 2.2.

Theorem 2.10. Suppose $f$ is a real valued function on $[a, b]$. Let $x_{0} \in[a, b]$. Suppose $f^{\prime}\left(x_{0}\right)$ exists. Let $G$ be a positive real valued function on an interval I which contains the range of $f$. Suppose $G$ is $m$-differentiable at the point $f\left(x_{0}\right)$. Let $H(t)=G(f(t))$, for all $t \in[a, b]$. Then $H$ is $m$-differentiable at $x_{0}$, and $H^{\mid}\left(x_{0}\right)=\left(G^{\mid}\left(f\left(x_{0}\right)\right)^{f^{\prime}\left(x_{0}\right)}\right.$.

Proof. By Lemma 2.4,

$$
\begin{aligned}
\left(G^{\mid}\left(f\left(x_{0}\right)\right)^{f^{\prime}\left(x_{0}\right)}\right. & =\left(\exp \left(\frac{d \log G\left(f\left(x_{0}\right)\right)}{d y}\right)\right)^{\frac{d f\left(x_{0}\right)}{d x}}(\text { with } y \in I) \\
& =\exp \left(\frac{d f\left(x_{0}\right)}{d x} \frac{d(\log G) f\left(x_{0}\right)}{d y}\right) \\
& =\exp \left(\frac{d((\log G) \circ f)\left(x_{0}\right)}{d x}\right) \\
& =\frac{D H\left(x_{0}\right)}{D x} .
\end{aligned}
$$

Another Proof:

Use Proposition 2.8 and the relation

$$
\left(\frac{H(x)}{H\left(x_{0}\right)}\right)^{\frac{1}{x-x_{0}}}=\left(\left(\frac{G(f(x))}{G\left(f\left(x_{0}\right)\right)}\right)^{\frac{1}{f(x)-f\left(x_{0}\right)}}\right)^{\frac{f(x)-f\left(x_{0}\right)}{x-x_{0}}}
$$

for the case $f(x) \neq f\left(x_{0}\right)$. Separate the case $f(x)=f\left(x_{0}\right)$. 
Lemma 2.11. Let $F$ be a positive real valued function defined on $[a, b]$. If $F$ has a local maximum at a point $x_{0}$ in $(a, b)$ and if $\frac{D F\left(x_{0}\right)}{D x}$ exists, then $\frac{D F\left(x_{0}\right)}{D x}=1$.

Proof. Suppose $\frac{D F\left(x_{0}\right)}{D x}$ exists. Then $\frac{d \log F\left(x_{0}\right)}{d x}$ exists. Suppose $F$ has a local maximum at $x_{0}$ in $(a, b)$. Then $\frac{d \log F\left(x_{0}\right)}{d x}=0$, and hence $\frac{D F\left(x_{0}\right)}{D x}=\exp \left(\frac{d \log F\left(x_{0}\right)}{d x}\right)=1$.

Direct Proof: $\left(\frac{F(x)}{F\left(x_{0}\right)}\right)^{\frac{1}{x-x_{0}}} \geq 1$, if $x<x_{0}$, and $\left(\frac{F(x)}{F\left(x_{0}\right)}\right)^{\frac{1}{x-x_{0}}} \leq 1$, if $x>x_{0}$, when $x$ is in a suitable neighborhood of $x_{0}$.

Theorem 2.12. Suppose $F$ and $G$ are continuous positive real valued functions on $[a, b]$ which are $m$-differentiable in (a, $b)$. Then there is $a$ point $x_{0}$ in $(a, b)$ such that $\left(\frac{F(b)}{F(a)}\right)^{\frac{G^{\prime}\left(x_{0}\right)}{G\left(x_{0}\right)}}=\left(\frac{G(b)}{G(a)}\right)^{\frac{F^{\prime}\left(x_{0}\right)}{F\left(x_{0}\right)}}$

Proof. Use Theorem 5.9 in [23], and Lemma 2.4 to find a point $x_{0}$ in $(a, b) \operatorname{such} \operatorname{that}(\log F(b)-\log F(a)) \frac{G^{\prime}\left(x_{0}\right)}{G\left(x_{0}\right)}=(\log G(b)-$ $\log G(a)) \frac{F^{\prime}\left(x_{0}\right)}{F\left(x_{0}\right)}$

Proposition 2.13. Suppose $F$ is a continuous positive real valued function on $[a, b]$ which is $m$-differentiable in $(a, b)$. Then $\left(\frac{F(b)}{F(a)}\right) \frac{1}{b-a}=$ $\frac{D F\left(x_{0}\right)}{D x}$, for some $x_{0} \in(a, b)$.

Proof. By the mean value theorem, there is a point $x_{0} \in(a, b)$ such that $\log F(b)-\log F(a)=(b-a) \frac{d \log F\left(x_{0}\right)}{d x}$.

Theorem 2.14. Suppose $F$ is an $m$-differentiable positive real valued function on $(a, b)$.

(a) If $F^{\mid}(x) \geq 1$ for all $x \in(a, b)$, then $F$ is monotonically increasing in $(a, b)$.

(b) If $F^{\mid}(x)=1$ for all $x \in(a, b)$, then $F$ is a constant function in $(a, b)$.

(c) If $F^{\mid}(x) \leq 1$ for all $x \in(a, b)$, then $F$ is monotonically decreasing in $(a, b)$.

Proof. Apply Theorem 5.11 in [23] to $\log F$ function.

Theorem 2.15. Suppose $F$ is an $m$-differentiable positive real valued function on $[a, b]$. Let $\lambda$ be a constant such that $F^{\mid}(a)<\lambda<F^{\mid}(b)$. Then there is a point $x_{0}$ in $(a, b)$ such that $F^{\mid}\left(x_{0}\right)=\lambda$.

Proof. Observe that $\exp \left(\frac{d \log F(a)}{d x}\right)<\lambda<\exp \left(\frac{d \log F(b)}{d x}\right)$ and hence $\frac{d \log F(a)}{d x}<\log \lambda<\frac{d \log F(b)}{d x}$. By Theorem 5.12 in [23], there is a $x_{0} \in(a, b)$ such that $\frac{d \log F\left(x_{0}\right)}{d x}=\log \lambda$. In this case, $F^{\mid}\left(x_{0}\right)=\lambda$.

Theorem 2.16. Let $F$ be a positive real valued function on $[a, b]$, and $n$ be a positive integer such that $\frac{D^{n-1} F(t)}{D x^{n-1}}$ is continuous on $[a, b]$ and $\frac{D^{n} F(t)}{D x^{n}}$ exists for every $t \in(a, b)$. Let $\alpha, \beta$ be points in $[a, b]$. Then there is a point $x_{0}$ between $\alpha$ and $\beta$ such that $\frac{F(\beta)}{F(\alpha)}=\sum_{k=1}^{n-1}\left(\frac{D^{k} F(\alpha)}{D x^{k}}\right)^{\frac{(\beta-\alpha)^{k}}{k !}}+\left(\frac{D^{n} F\left(x_{0}\right)}{D x^{n}}\right)^{\frac{(\beta-\alpha)^{n}}{n !}}$

Proof. By Remark 2.7, $\frac{d^{n-1} \log F}{d x^{n-1}}$ is continuous on $[a, b]$ and $\frac{d^{n} \log F(t)}{d x^{n}}$ exists for every $t \in(a, b)$. By Theorem 5.15 in $[23]$, there is a point $x_{0}$ between $\alpha$ and $\beta$ such that $f(\beta)-f(\alpha)=\sum_{k=1}^{n-1} \frac{d^{k} f(\alpha)}{d x^{k}} \frac{(\beta-\alpha)^{k}}{k !}+\frac{d^{n} f\left(x_{0}\right)}{d x^{n}} \frac{(\beta-\alpha)^{n}}{n !}$, where $f=\log F$. The result follows from this relation.

\section{Multiplicative Riemann integration}

Definition 3.1. Let $[a, b]$ be a given interval. A partition $P=x_{0}, x_{1}, \ldots, x_{n}$ of $[a, b]$ satisfies $a=x_{0} \leq x_{1} \leq \ldots \leq x_{n-1} \leq x_{n}=b$. Let $D$ be the collection of all partitions of $[a, b]$. This collection $D$ is a directed set, directed by a relation $\leq$ defined by: $P_{1} \leq P_{2}$ if and only if $P_{1} \subseteq P_{2}$. Let $F:[a, b] \rightarrow(0, \infty)$ be a function such that $m \leq F(x) \leq M$, for some $m>0$ and $M<\infty$, for all $x \in[a, b]$. To each partition $P=x_{0}, x_{1}, \ldots, x_{n}$ of $[a, b]$, fix $t_{i} \in\left[x_{i-1}, x_{i}\right]$, for $i=1,2, \ldots, n$, define $F_{P}=\prod_{i=1}^{n} F\left(t_{i}\right)^{\left(x_{i}-x_{i-1}\right)}$. Suppose all nets $\left(F_{P}\right)_{P \in D}$ converge uniformly to a common number $p \in(0, \infty)$ in the following sense: For every $\varepsilon>0$, there is a partition $P_{0}$ of $[a, b]$ such that $\left|F_{P}-p\right|<\varepsilon$, for all partitions $P \geq P_{0}$ in $D$, and for all selections of $t_{i}$. The number $p$ is called the $m$-Riemann integral of $F$ of $[a, b]$, and it is denoted by $M_{a}^{b} F(x) D x$, or simply, $M_{a}^{b} F$. In this case, let us say that $F$ is m-Riemann integrable over $[a, b]$.

Remark 3.2. Let $F$ be as in Definition 3.1. Then $F$ is m-Riemann integrable over $[a, b]$ if and only if log $F$ is Riemann integrable over $[a, b]$. Moreover,

$$
M_{a}^{b} F(x) D x=\exp \int_{a}^{b} \log F(x) d x
$$

in this case. Let $f$ be a bounded real valued function on $[a, b]$. Then $f$ is Riemann integrable over $[a, b]$ if and only if $\exp f$ is $m$-Riemann integrable over $[a, b]$. Moreover,

$$
\int_{a}^{b} f(x) d x=\log M_{a}^{b} \exp f(x) D x
$$


in this case.

Lemma 3.3. Let $F:[a, b] \rightarrow(0, \infty), G:[a, b] \rightarrow(0, \infty)$ be functions which are m-Riemann integrable over $[a, b]$. Then the pointwise multiplication function $F G:[a, b] \rightarrow(0, \infty)$ is also m-Riemann integrable over $[a, b]$, and $M_{a}^{b}(F G)(x) D x=\left(M_{a}^{b} F(x) D x\right)\left(M_{a}^{b} G(x) D x\right)$. If $F(x)=c>0$, for every $x \in[a, b]$, then $M_{a}^{b} F(x) D x=c^{b-a}$.

Proof. It follows from Definition 3.1.

Let us recall that for every number $x \in(0, \infty),|x|_{\times}=\max \left\{x, x^{-1}\right\}$, and for every $x, y \in(0, \infty),|x y|_{\times} \leq|x|_{\times}|y|_{\times}$. Direct verification is applicable.

Lemma 3.4. Let $F:[a, b] \rightarrow(0, \infty)$ be m-Riemann integrable. Then, $\left|M_{a}^{b} F(x) D x\right|_{\times} \leq M_{a}^{b}|F(x)|_{\times} D x$.

Proof. It follows from Definition 3.1.

Theorem 3.5. Suppose $f:[a, b] \rightarrow(0, \infty)$ be $m$-Riemann integrable. For $a \leq x \leq b$, define $F(x)=M_{a}^{x} f(t) D t$. Then, $F$ is continuous on $[a, b]$. Moreover, if $f$ is continuous at a point $x_{0}$ of $[a, b]$, then $F$ is $m$-differentiable at $x_{0}$, and $F^{\mid}\left(x_{0}\right)=f\left(x_{0}\right)$.

Proof. $\quad \int_{a}^{x} \log f(t) d t$ is a continuous function of $x$ in $[a, b]$, by Theorem 6.20 in [23] and Remark 3.2. Thus, $F(x)=\exp \int_{a}^{x} \log f(t) d t$ is a continuous function of $x$ in $[a, b]$. Also, if $f$ is continuous at a point $x_{0}$ of $[a, b]$, then $\left.\frac{d \int_{a}^{x} \log f(t) d t}{d x}\right|_{x=x_{0}}=\log f\left(x_{0}\right)$, by Theorem 6.20 in [12]. Thus, $F^{\mid}\left(x_{0}\right)=f\left(x_{0}\right)$, when $f$ is continuous at $x_{0}$.

Theorem 3.6. Let $f$ be an m-Riemann integrable function on $[a, b]$. Suppose there is an m-differentiable function $F$ on $[a, b]$ such that $F^{\mid}=f$. Then, $M_{a}^{b} f(x) D x=\frac{F(b)}{F(a)}$.

Proof. The function $\log f$ is Riemann integrable over $[a, b]$. The relation $F^{\mid}=f \operatorname{implies}$ that $\exp \frac{d \log F}{d x}=f$ or $\frac{d \log F}{d x}=\log f$. By Theorem 6.21 in [23], $\int_{a}^{b} \log f(x) d x=\log F(b)-\log F(a)$, and hence $M_{a}^{b} f(x) D x=\frac{F(b)}{F(a)}$.

\section{Multiplicative abstract measure integration}

Definition 4.1. Let $(X, \mathfrak{M}, \mu)$ be a positive measure space. See [24]. Let $s: X \rightarrow[1, \infty)$ be a simple measurable function and let $s=\sum_{i=1}^{n} \alpha_{i} \chi_{A_{i}}$, where $\alpha_{i}$ are distinct real numbers and $A_{i}$ are pairwise disjoint sets. For $A \in \mathfrak{M}$, define $M_{A} s D \mu=\Pi_{i=1}^{n} \alpha_{i}{ }^{\mu}\left(A \cap A_{i}\right)$. It is $+\infty$ if and only if some $\alpha_{i}>1$ with $\mu\left(A \cap A_{i}\right)=+\infty$. Let $f: X \rightarrow[1, \infty]$ be a measurable function. For $A \in \mathfrak{M}$, define $M_{A} f D \mu=\sup _{1<s}<f M_{A}$ sD $\mu$, where s represents a simple measurable function. Let us say that $f: X \rightarrow(0, \infty)$ is $m$-absolutely $m$-integrable, if $M_{A}|f|_{\times} D \mu<\infty$. To each measurable $f: X \rightarrow(0, \infty)$, let us define $f_{\times}^{+}: X \rightarrow[1, \infty)$, and $f_{\times}^{-}: X \rightarrow[1, \infty)$ by $f_{\times}^{+}(x)=\max \{1, f(x)\}$, and $f_{\times}^{-}(x)=\max \left\{1, f(x)^{-1}\right\}$, for every $x \in X$. Then $|f|_{\times}=f_{\times}^{+} f_{\times}^{-}$, and $f=\frac{f_{\times}^{+}}{f_{\times}^{-}}$. If $f$ is absolutely $m$-integrable over $X$, then let us define $M_{A} f D \mu=\frac{M_{A} f_{\times}^{+} D \mu}{M_{A} f_{\times}^{-} D \mu}$, for every $A \in \mathfrak{M}$.

Remark 4.2. If $f \in L^{1}(\mu)$ and $f$ is real valued, then $\exp f$ is $m$-absolutely $m$-integrable on $X$, and $\log M_{X} \exp f D \mu=\int_{X} f d \mu$. If $F$ is $m$-absolutely m-integrable on $X$, then $\log F \in L^{1}(\mu)$ and $M_{X} F D \mu=\exp \int_{X} \log F d \mu$.

Theorem 4.3. Let $\left(f_{n}\right)_{n=1}^{\infty}$ be a sequence of measurable functions on $X$ and $f$ be a measurable function $X$ such that

(a) $1 \leq f_{1}(x) \leq f_{2}(x) \leq \ldots \leq \infty$, for every $x \in X$, and

(b) $f_{n}(x) \rightarrow f(x)$ as $n \rightarrow \infty$, for every $x \in X$.

Then $M_{X} f_{n} D \mu \rightarrow M_{X} f D \mu$, as $n \rightarrow \infty$.

Proof. Apply the classical monotone convergence theorem to the functions $\log f_{n}$ and $\log f$ and use Remark 4.2 .

Corollary 4.4. Let $f_{n}: X \rightarrow[1, \infty]$ be measurable for $n=1,2, \ldots$, and let $f(x)=\prod_{n=1}^{\infty} f_{n}(x)$, for every $x \in X$. Then $M_{X} f D \mu=$ $\prod_{n=1}^{\infty} M_{X} f_{n} D \mu$.

Proof. Observe that $M_{X} f_{1} f_{2} D \mu=\left(M_{X} f_{1} D \mu\right)\left(M_{X} f_{2} D \mu\right)$. Extend this relation to finite products $f_{1} f_{2} \ldots f_{n}$. Now, apply the previous Theorem 4.3 .

The following proposition can be verified directly.

Proposition 4.5. Let $f, g$ be $m$-absolutely $m$-integrable positive real valued functions on $X$. Let $c>0$ be a scalar. Then $M_{X} f g D \mu=$ $\left(M_{X} f D \mu\right)\left(M_{X} g D \mu\right)$ and $M_{X} f^{c} D \mu=\left(M_{X} f D \mu\right)^{c}$.

Proposition 4.6. Let $f_{n}: X \rightarrow[1, \infty]$ be measurable, for each $n=1,2, \ldots$, Then

$$
M_{X} \lim \inf _{n \rightarrow \infty} f_{n} D \mu \leq \lim \inf _{n \rightarrow \infty} M_{X} f_{n} D \mu .
$$

Proof. Apply the classical Fatou's lemma to the functions $\log f_{n}$ and use Remark 4.2.

Theorem 4.7. Suppose $\left(f_{n}\right)_{n=1}^{\infty}$ is a sequence of positive real valued measurable functions on $X$ such that $f(x)=\lim _{n \rightarrow \infty} f_{n}(x)$ for some $f(x)>0$, for every $x \in X$. Suppose there is an m-integrable function $g: X \rightarrow[1, \infty)$ such that $\left|f_{n}\right|_{\times} \leq g(x), \forall n=1,2, \ldots, \forall x \in X$. Then $f$ is an $m$-absolutely integrable function, $\lim _{n \rightarrow \infty} M_{X}\left|\frac{f_{n}}{f}\right|_{\times} D \mu=1$, and $\lim _{n \rightarrow \infty} M_{X} f_{n} D \mu=M_{X} f D \mu$. 
Proof. Let $F_{n}=\log f_{n}$ and $F=\log f$. Let $G=\log g$. Then $\lim _{n \rightarrow \infty} F_{n}(x)=F(x), \forall x \in X$, and $G \in L^{1}(\mu)$. Also, $\left|F_{n}(x)\right|=\left|\log f_{n}(x)\right|=$ $\log \left|f_{n}\right|_{\times} \leq \log g=G, \forall x \in X$. By the classical dominated convergence theorem, $\int_{X} F_{n} d \mu \rightarrow \int_{X} F d \mu$ and $\lim _{n \rightarrow \infty} \int_{X}\left|F_{n}-F\right| d \mu=0$, as $n \rightarrow \infty$. Then $\exp \int_{X} \log f_{n} d \mu \rightarrow \exp \int_{X} \log f d \mu$, as $n \rightarrow \infty$, and $\lim _{n \rightarrow \infty} \exp \int_{X}\left|\log \left(\frac{f_{n}}{f}\right)\right| d \mu=\lim _{n \rightarrow \infty} \exp \int_{X} \log \left|\frac{f_{n}}{f}\right|_{\times} d \mu=1$. Thus, $\lim _{n \rightarrow \infty} M_{X} f_{n} D \mu=M_{X} f D \mu$, and $\lim _{n \rightarrow \infty} M_{X}\left|\frac{f_{n}}{f}\right|_{\times} D \mu=1$.

Proposition 4.8. Suppose $f: X \rightarrow(0, \infty)$ is an m-absolutely m-integrable function. Then $\left|M_{X} f D \mu\right|_{\times} \leq M_{X}|f|_{\times} D \mu$.

Proof. It follows from definitions.

Proposition 4.9. (a) Let $f: X \rightarrow[1, \infty]$ be a measurable function on $X$ such that $M_{X} f D \mu=1$. Then $f=1$ almost everywhere on $X$. (b) Let $f: X \rightarrow(0, \infty)$ be $m$-absolutely m-integrable on $X$. Suppose $M_{E} f D \mu=1, \forall E \in \mathfrak{M}$. Then $f=1$ almost everywhere on $X$. (c) Let $f: X \rightarrow(0, \infty)$ be m-absolutely m-integrable on $X$. Suppose $\left|M_{X} f D \mu\right|_{\times}=M_{X}|f|_{\times} D \mu$. Then there is a constant $\alpha$ such that $\alpha f=|f|_{\times}$almost everywhere on $X$.

Proof. Use Proposition 1.3 along with Theorem 1.39 in [24] for $\log f$.

\section{Conclusions}

Theorem 11.33 in [23] states that a Lebesgue integrable bounded real valued function on $[a, b]$ is Riemann integrable over the interval if and only if the function is continuous almost everywhere in that interval with respect to classical Lebesgue measure. One can state a corresponding result for m-Riemann integrable functions. Such observations may give a hope to transform every known result in additive calculus and abstract measure integration theory through logarithmic exponential transformations. But, it is not true. Theorems 2.10 and 2.12 reveal difficulties in transforming chain rule and a generalized mean value theorem which is used for establishing L'Hospital's rules. It is yet to be tried for replacement of exponential transformation and its inverse logarithmic transformation by means of other transformations like Lorentz transformation and its inverse Lorentz transformation of the theory of Einstein's special relativity. It is yet to be tried to extend results which provide generalizations of research work in differentiation, integration and measure theory like the ones provided in [25]-[30].

\section{Acknowledgement}

Dr. C. Ganesa Moorthy (Professor, Department of Mathematics, Alagappa University, Karaikudi- 630003, INDIA) gratefully acknowledges the joint financial support of RUSA-Phase 2.0 grant sanctioned vide letter No.F 24-51/2014-U, Policy (TN Multi-Gen), Dept. of Edn. Govt. of India, Dt. 09.10.2018, UGC-SAP (DRS-I) vide letter No.F.510/8/DRS-I/2016 (SAP-I) Dt. 23.08.2016 and DST (FIST - level I) 657876570 vide letter No.SR/FIST/MS-I/2018-17 Dt. 20.12.2018.

\section{References}

[1] A. E. Bashirov, E. M. Kurpinar, A. Ozyapici, Multiplicative calculus and its applications, J. Math. Anal. Appl., 337 (2008), $36-48$.

[2] C. G. Moorthy, Infinite products using multiplicative modulus function, Math. Student, 88(3\&4) (2019), 39-54.

[3] M. Grossman, R. Katz, Non-Newtonian Calculus, Les Press, Pigeon Cove, MA, 1972.

[4] U. Kadak, M. Ozlük, Generalized Runge-Kutta method with respect to nonNewtonian calculus, Abst. Appl. Anal., (2015), Article ID 594685, 10 pages.

[5] A. Uzer, Multiplicative type complex calculus as an alternative to the classical calculus, Comput. Math. Appl., 60 (2010), $2725-2737$.

[6] N. Yalcin, E. Celik, A. Gokdogan, Multiplicative Laplace transform and its applications, Optik, 127(20) (2016), 9984-9995.

[7] T. Abadeljawad, On multiplicative fractional calculus, (2015), arXiv:1510.04176v1[math.CA].

[8] D. Aniszewska, Multiplicative Runge-Kutta method, Non-Linear Dyn., 50(2007), 265-272.

[9] A. E. Bashirov, E. Misirli, Y. Tandogdu, A. Ozyapici, On modelling with multiplicative differential equations, Appl. Math. J. Chinese Univ., 26(4)(2011), $425-428$.

[10] A. E. Bashirov, M. Riza, Complex multiplicative calculus, (2011), arXiv:1103.1462[math.CV].

[11] A. E. Bashirov, M. Riza, On complex multiplicative differentiation, TWMS J. App. Eng. Math., 1(1) (2011)75-85.

[12] A. E. Bashirov, E. M. Kurpinar, A. Ozyapici, Multiplicative Calculus and its applications, J. Math. Anal. Appl., 337 (2008), $36-48$.

[13] A. E. Bashirov, S. Norozpour, On an alternative view to complex calculus, Math. Meth. Appl. Sci., 41 (2018), 7313-7324.

[14] A. H. Bhat, J. Majid, I. A. Wani, Multiplicative Sumudu transform and its applications, J. Emerging Tech. Innovative Res., 6(1)(2019), 579-589.

[15] K. Boruah, B. Hazarika, Application of geometric calculus in numerical analysis and difference sequence spaces, J. Math. Anal. Appl., 449(2)(2017), $1265-1285$.

[16] K. Boruah, B. Hazarika, G-Calculus, TWMS J. Appl. Eng. Math., 8(1) (2018), 94-105.

[17] K. Boruah, B. Hazarika, Bigeometric integral calculus, TWMS J. Appl. Eng. Math., 8(2) (2018), 374-385.

[18] K. Boruah, B. Hazarika, A. E. Bashirov, Solvability of bigeometric differential equations by numerical methods, Bol. Soc. Paran. Mat. (in press).

[19] D. Filip, C. Piatecki, An overview on the non-Newtonian calculus and its applications to economics, Appl. Math. Comput., 187(1) (2007), 68-78.

[20] L. Florack, H. Assen, Multiplicative calculus in biomedical image analysis, J. Math. Imaging Vis., 42 (2012), 64-75.

[21] D. Stanley, A multiplicative calculus, Primus, 9(4) (1999), 310-326.

[22] E. J. P. G. Schmidt, On multiplicative Lebesgue integration and families of evolution operators, Math. Scand., 29 (1971), 113-133.

[23] W. Rudin, Principles of Mathematical Analysis, Third edition, McGraw Hill, London, 1976.

[24] W. Rudin, Real and Complex Analysis, Third edition, McGraw Hill, New York, 1987.

[25] N. Marikkannan, P. Sooriyakala, C. G. Moorthy, Certain applications of differential subordination and superordination, Int. J. Pure Appl. Math., 34(4) (2007), 547-558.

[26] N. Marikkannan, C. G. Moorthy, On applications of differential subordination and superordination, Tamkang J. Math., 39(2) (2008), $155-164$.

[27] C. G. Moorthy, Measure theory and Hausdorff dimension of Cantor sets of Continued fractions, Ph.D. Thesis, Alagappa University, 1992.

[28] C. G. Moorthy, N. Marikkannan, M. P. Jeyaraman, Applications of differential subordination and superordination, J. Indones. Math. Soc., 14(1) (2012),

[29] C. G. Moorthy, R. Vijaya, P. Venkatachalapathy, Hausdorff dimension of Cantor-like sets, Kyungpook Math. J., 32(2) (1992), 197-202.

[30] C. G. Moorthy, A problem of good on Hausdorff dimension, Mathematika, 39(2) (1992), 244-246. 